\title{
Formation of Pt nanorods on nanoporous anodic aluminum oxides by controlled nucleation sites
}

\author{
Kai-Tze Huang a , Po-Cheng Kuo ${ }^{\text {a }}$, Yeong-Der Yao ${ }^{\text {b,* }}$ \\ a Institute of Materials Science and Engineering, National Taiwan University, Taipei 10617, Taiwan, ROC \\ b Department of Materials Engineering, Tatung University, Taipei 10452, Taiwan, ROC
}

\section{A R T I C L E I N F O}

\section{Article history:}

Received 31 January 2008

Received in revised form 10 October 2008

Accepted 4 November 2008

Available online $\mathrm{xxxx}$

\section{Keywords:}

Anodic aluminum oxide

Nanorod

Patterning

Platinum nanostructures

Sputtering

Scanning electron microscopy

\begin{abstract}
A B S T R A C T
Vertical-aligned Pt nanorods are fabricated on the surface of nanoporous anodic aluminum oxide (AAO) templates by magnetron sputtering. The size effect of AAO templates, which is focused on in this study, would influence the morphology of stacked atoms. The rim of the pores, which act as obstacles to the stacked atoms, could prevent them from forming continuous films. The continuous films are commonly formed because of the larger interpore distance which is compared to the grain size of stacked atoms. Between closely-distributed pores, the shorter interpore distance, namely smaller surface area, indicates insufficient surface energy for heterogeneous nucleation, restricts the growth of stacked atoms. Nevertheless, there still remain regions with larger interpore distance. On these constricted regions, instead of continuous films, isolated nanorods are formed. In this study, a nanorod growth diagram is deduced, and understanding of the growth of nanorods on AAO templates is furthered.
\end{abstract}

(C) 2008 Elsevier B.V. All rights reserved.

\section{Introduction}

The preparation of nano-sized materials such as nanodots [1-10], nanowires or nanorods [11-21] has attracted much attention in recent years. Anodic aluminum oxide (AAO) templates possess a well-aligned nano-sized porous structure which can be provided for fabricating nano-sized materials. Research on AAO templates in recent years has focused on: (1) filling the pores of AAO templates with materials [8,11-17,21,22], (2) using AAO templates as deposition or etch masks [7,9,10,23-25], (3) inducing surface structural growth [18], and (4) examining deposition on the modified structure of AAO templates $[19,20]$. Most of the research has focused on the interiors of the pores of AAO templates, but the formation mechanisms that occur outside the pores, on the AAO template surface, are not yet clear [18]. In this study, in order to investigate this mechanism, we directly deposit Pt atoms on the surface of an AAO template surface to observe how isolated nanorods are formed.

\section{Experiment}

Pt atoms were perpendicularly deposited on anodic aluminum oxide (AAO) templates by magnetron sputtering at room temperature with a deposition rate of about $8 \mathrm{~nm} / \mathrm{min}$ and a sputtering pressure of about $5 \times 10^{-3}$ Torr. The distance between target and AAO template is

\footnotetext{
* Corresponding author.

E-mail address: ydyao@phys.sinica.edu.tw (Y.-D. Yao).
}

about $5 \mathrm{~cm}$. Before deposition, surface defects of the AAO templates were removed by $\mathrm{Ar}^{+}$ion bombardment. The morphologies of the stacked atoms were identified by a Hitachi S-4200 scanning electron microscope (SEM) with operating voltage of $15 \mathrm{kV}$. The grain size and structure of Pt were identified by a Philips PW3040/60 X-ray diffraction (XRD) with a Cu-K $\mathrm{K}_{\alpha}$ radiation source $(\lambda=1.54060 \AA)$.

AAO template surfaces were used to provide the nucleation and growth sites for the formation of Pt nanorods. Fig. 1 shows the schematic illustration of the interpore region and corner region, the sizes of which we defined as $\lambda$ and $\delta$, respectively. In order to examine the effect of pore diameter $(\varphi)$ and interpore size $(\lambda)$ of AAO templates on the growth mechanism of Pt, four types of AAO templates with pore diameters of approximately 24, 75, 133, and $206 \mathrm{~nm}$ were used. Here, we define these templates as AAO24, AAO75, AAO133, and AAO 206 , respectively. In Fig. 1, the corner region size $(\delta)$ is given by

$$
\begin{aligned}
\delta & =\left[(\varphi+\lambda) \times \frac{\sqrt{3}}{2} \times \frac{2}{3}-\frac{\varphi}{2}\right] \times 2 \\
& \approx 0.155 \varphi+1.155 \lambda .
\end{aligned}
$$

\section{Results and discussion}

Fig. 2(a) and (b) shows SEM images of the plane view of AAO75 and AAO206. It can be seen that $\lambda$ and $\delta$ have increased with the increase in the pore diameter. The white or light gray region between the pores of the AAO template surface is the area we focus on in this study. After Pt deposition, almost all the Pt atoms are stacked on the surfaces of the AAO templates, as shown in Figs. 3 and 4. Fig. 3 shows a SEM image of 


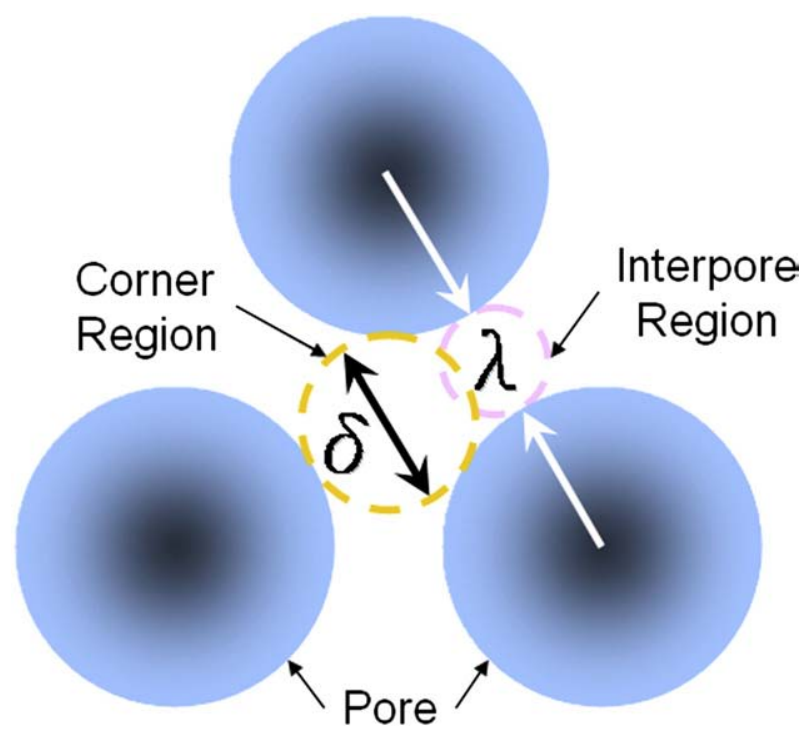

Fig. 1. The schematic illustration of the interpore region size $\lambda$ and corner region size $\delta$.

Pt nanorods grown on AAO75 by deposition of Pt for only $375 \mathrm{~s}$. The white dots in Fig. 3(a) are the Pt nanorods. The heights of these nanorods were determined by two methods, observation of SEM images and calculation by deposition rate, with almost identical results of about $50 \mathrm{~nm}$. Amazingly, the Pt nanorods have mainly grown on the corner regions of the AAO templates. With an increase of the deposition time, the Pt nanorods grow further by increasing in both diameter and length, as shown in Fig. 3(b) and (c). The specific surface regions (corner regions) provide the nucleation sites for Pt grains, which proceed to grow into the form of nanorods. This nanorod growth behavior is introduced with a schematic illustration in Fig. 3(d). Fig. 3(c) shows the results of increasing the deposition time to $1500 \mathrm{~s}$. In the bottom part of the image can be seen a section from which the Pt nanorods that formed on the AAO template have been removed. The length of the Pt nanorods is about $200 \mathrm{~nm}$. It can also be seen that some vacant spaces exist between the Pt nanorods, and the nanorods are almost completely isolated from each other. The lengths of nanorods increase with longer deposition times, but the diameters are restricted to the periodic distance of the neighboring pores. Because the Pt atoms not only stack up on the top of the nanorods but also fill in the vacant spaces, the diameters of the nanorods increase until adjacent nanorods come into contact with each other $[19,20]$. The average diameter of the nanorods is $84 \mathrm{~nm}$. Nanorods of magnetic materials formed in this manner would be useful for ultra-high density magnetic recording media applications $[1-4,6-9,13,21]$. If one rod is equal to one recording bit, then the recording density of a magnetic material made in this manner would be about $70 \mathrm{Gbit} / \mathrm{in}^{2}$.

By the same fabrication process, AAO24, AAO133, and AAO206 were also used to deposit Pt atoms, with results that differed from those with deposition on AAO75. Fig. 4(a) and (b) shows the SEM images of Pt atoms on AAO24. Fig. 4(a) shows the results of a deposition time of $375 \mathrm{~s}$. Calculated by deposition rate, the height of the stacked Pt atoms is about $50 \mathrm{~nm}$, but observation via SEM does not confirm that length, instead revealing a shorter length. Only a slightly greater amount of Pt atoms is stacked on the corner regions of AAO templates. As the deposition time is increased, the Pt atoms also stack on the corner regions to form nanorods, as shown in Fig. 4(b). Fig. 4(c) and (d) shows the SEM images of Pt atoms deposited on AAO206. Because of the larger interpore distance of the AAO206 surface, more Pt atoms have stacked on the surface, forming clusters. Pt clusters have tended to form at the surface edges around pores randomly and then to connect to each other to form Pt networks with increased deposition time is increased. Fig. 4(d) also shows a cross-section of AAO206 with 1500 s Pt deposition. We found that when Pt atoms are deposited on nanoporous AAO templates, they stack mainly on the interpore regions and corner regions and do not drop into the pores. As shown in Fig. 4(d), only small amounts of Pt atoms have dropped into the pores and adhered to the walls. It indicates that sputtering deposition is unlikely to result in the Pt atoms being deposited into the pores of AAO206, especially in the case of pores with smaller diameters (e.g., AAO24).

The growth mechanism of nanorods on the surfaces of AAO templates is attributed to the surface effect of high surface-to-volume ratios described in Ref. [18-20]. But this growth mechanism of nanorods on AAO templates cannot be used to quantitatively interpret why the atoms stacked only on specific regions. Theoretically, the majority of nucleation occurs heterogeneously at free surfaces, especially edges or defects. These are regions of higher surface free energy, and the excess energy becomes available for material nucleation. So, in order to reduce the surface free energy, heterogeneous nucleation occurs at the edges or defects. It is obvious that the Pt clusters nucleated and grew around the pores, as shown in Fig. 4(c). All the nucleation sites are conjointly formed. However, once the surface size drops below a critical value, while nucleation still occurs, the subsequent growth of the material is restricted. Thus, these
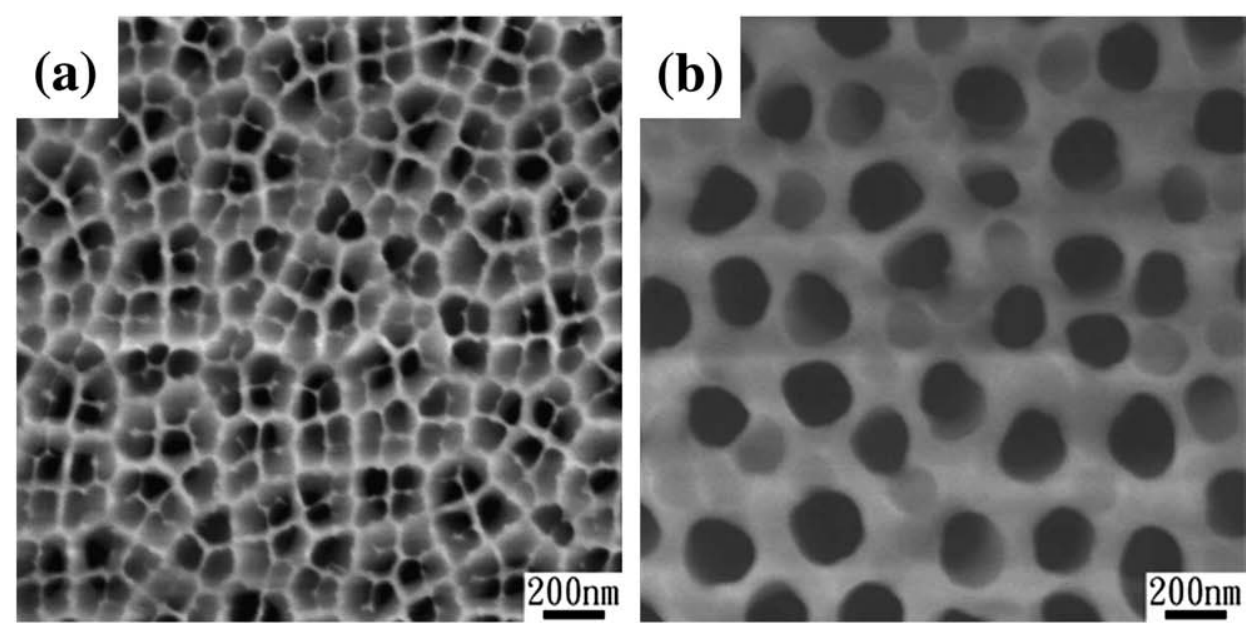

Fig. 2. SEM images of plane views of (a) AAO75 and (b) AAO206. 

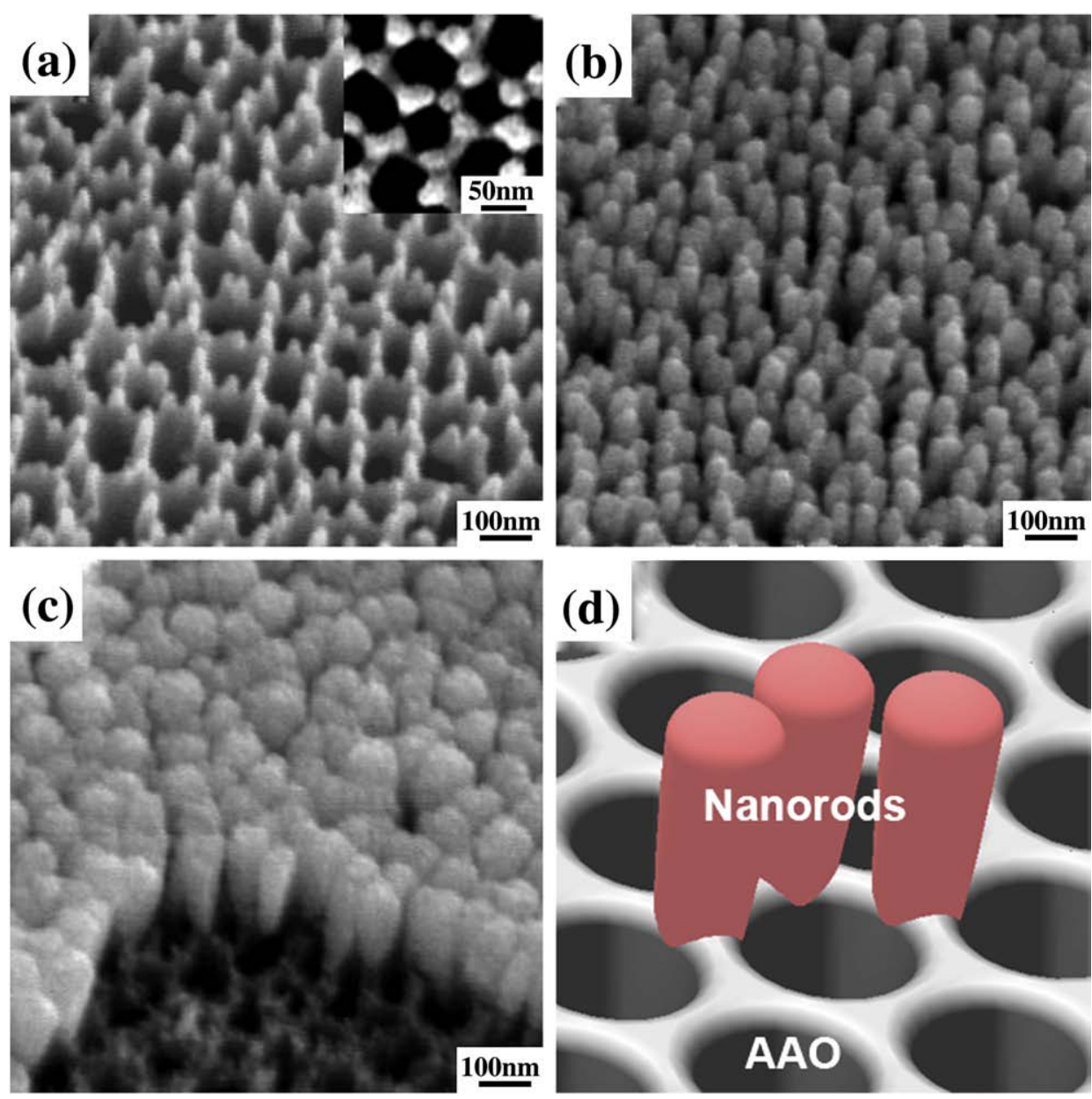

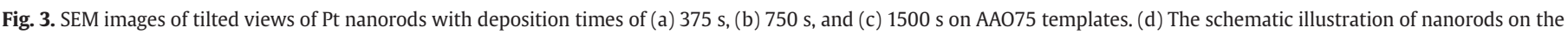
AAO template. The inset is the corresponding plane view of (a).

metastable clusters cannot grow further and decompose, decreasing the free energy. The critical size of Pt clusters is close to the grain size. XRD pattern was used to estimate the grain size of the Pt nanorods grown on AAO templates. Fig. 5 shows the XRD patterns of sapphire and AAO75 template with $1500 \mathrm{~s}$ Pt deposition. These two samples are all highly Pt(111) oriented, and the Pt grain sizes, as estimated by Scherrer's formula [26], are all about $10 \mathrm{~nm}$. The Pt has a face-centered cubic structure. Here, the metal element Pt is deposited on $\mathrm{Al}_{2} \mathrm{O}_{3}$ substrates. Because metal elements possess higher surface free energy (e.g., $\mathrm{Pt}(111)$ is $3290 \mathrm{erg} / \mathrm{cm}^{2}, \mathrm{Ni}(111)$ is $3250 \mathrm{erg} / \mathrm{cm}^{2}$, and $\mathrm{Cu}(111)$ is $2550 \mathrm{erg} / \mathrm{cm}^{2}$ ) than the $\mathrm{Al}_{2} \mathrm{O}_{3}$ substrates, the growth mode for $\mathrm{Pt}$ cluster on $\mathrm{Al}_{2} \mathrm{O}_{3}$ is Volmer-Weber type [27]. The selected restriction with smaller interpore regions is useful for forming isolated Pt clusters on the corner regions. The clusters which form on the corner regions obey the Volmer-Weber mode and grow until the clusters cover the corner regions completely. The shapes of the initial growth Pt clusters are restricted to circular dots by surface free energy and strain energy, so the diameters of these circular dots are determined by the corner region size, as shown in Fig. 3(a). Furthermore, deposited atoms prefer to stack upward to form nanorods, causing the nanorods to lengthen and coarsen.

From the mechanism mentioned above, a nanorod growth diagram is deduced, as shown in Fig. 6. The diagram is divided into three regions by two dashed lines drawn according to two equations. The measured value of the Pt grain size is $10 \mathrm{~nm}$, which is used to estimate what shapes are formed by stacked Pt atoms on different types of AAO templates. Nanorod growth is restricted to the corner region only when the interpore size is smaller than $10 \mathrm{~nm}$. Let $\lambda=10 \mathrm{~nm}$; this equation, drawn as the upper dashed line in Fig. 6 , is the boundary above which the nanorods do not form. Furthermore, the restriction mechanism may also restrict the growth of nanorods on the corner regions, if corner size is smaller than $10 \mathrm{~nm}$. Eq. (1) is rewritten as:

$\lambda=0.866 \delta-0.134 \varphi$.

Inserting $\delta=10 \mathrm{~nm}$ into Eq. (2), we can obtain:

$\lambda=8.66-0.134 \varphi$.

This equation, drawn as the lower dashed line in Fig. 6, is the other boundary. The shapes of deposited Pt formed by stacked atoms can be estimated easily with this diagram. This can be confirmed by the four types of AAO templates we used in this study. In region (I), the larger surface size $(\lambda>10 \mathrm{~nm})$ contributes to the continuous growth of Pt clusters, leading to the formation of networks (e.g., AAO133 and AAO206). In region (II), the surface size in the restricted range $(\lambda<10 \mathrm{~nm}, \delta>10 \mathrm{~nm})$, the Pt atoms are restricted to form isolated nanorods (e.g., AAO75). In region (III), the smaller surface size $(\lambda<10 \mathrm{~nm}, \delta<10 \mathrm{~nm})$ restricts the growth of nuclei ideally. From the point of energy, the corner regions possess higher surface free energy, so greater numbers of nuclei aggregate on these regions. These aggregated nuclei reduce the surface free energy, and then the Pt growth behavior proceeds. The 

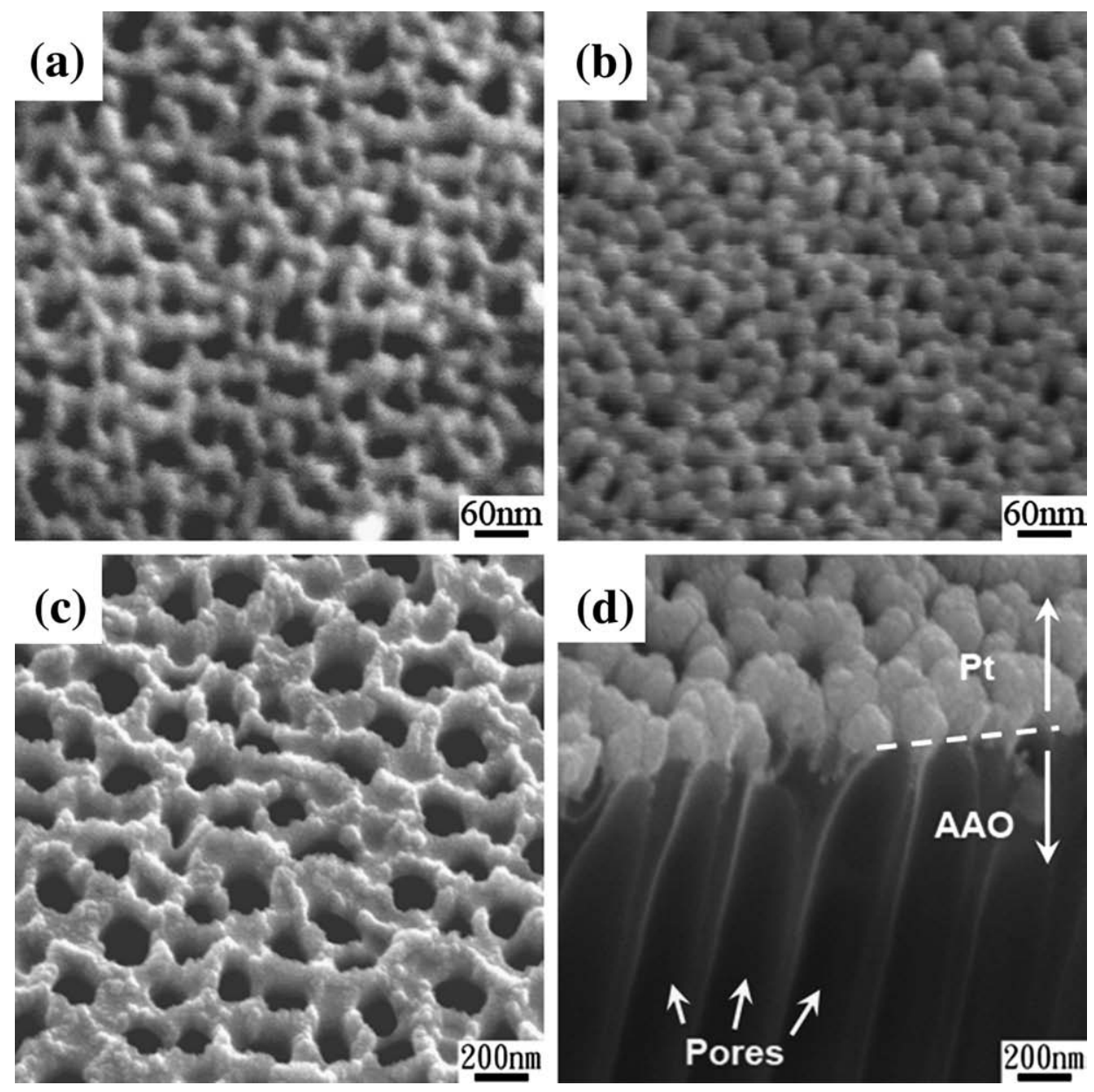

Fig. 4. SEM images of tilted views of Pt atoms with deposition times of (a) 375 s on AAO24, (b) 750 s on AAO24, (c) 375 s on AAO206, and (d) 1500 s on AAO206.

Pt nanorods are also formed on corner regions, but the isolation is less obvious. (e.g., AAO24).

\section{Conclusions}

We have fabricated isolated, vertical-aligned nanorods on AAO templates and analyzed their growth mechanisms. The Pt grain size,

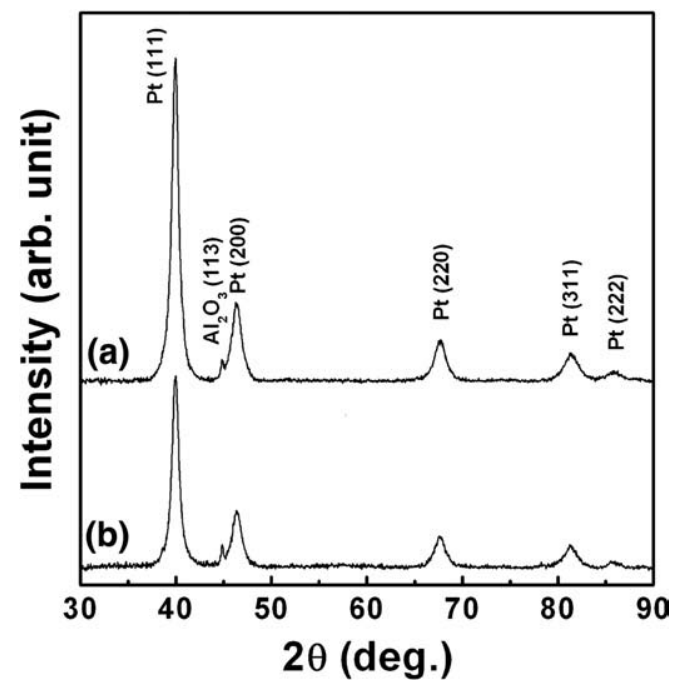

Fig. 5. XRD patterns of (a) sapphire and (b) AAO75 template with $1500 \mathrm{~s}$ Pt deposition. estimated by Scherrer's formula, is about $10 \mathrm{~nm}$. On AAO75, which has larger corner regions and smaller interpore regions than the Pt grain size, deposited Pt atoms are restricted to these larger corner regions and form nanorods. On AAO133 and AAO206, which have corner regions and interpore regions that are both larger than the Pt grain size, since the probabilities of Pt atoms being deposited on these regions are almost equivalent, Pt networks (or continuous films) are

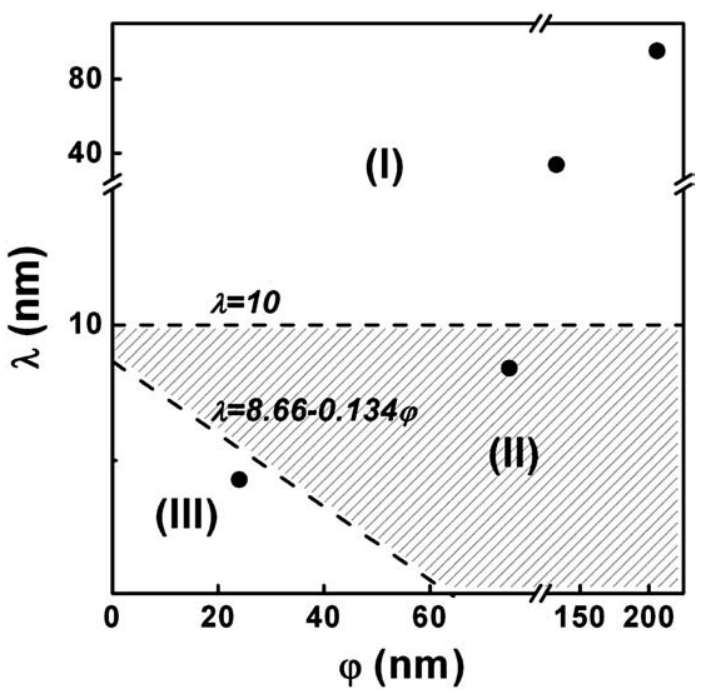

Fig. 6. Dependence of interpore size $\lambda$ with the pore diameter $\varphi$ of AAO templates; the four types of AAO templates are shown by solid circles. 
formed by continuous Pt clusters. On AAO24, which has corner regions and interpore regions that are both smaller than the Pt grain size, the Pt nanorods are also formed on the corner regions, but the isolation is less obvious. The resulting shorter Pt nanorods are formed by the aggregated nuclei. The growth mechanism in this study, as the material of these nanorods which is exchanged with magnetic element, is useful for the application of magnetic recording media.

\section{Acknowledgements}

This work is supported by the National Science Council of Taiwan and Ministry of Economic Affairs of Taiwan through Grant Nos. NSC 952112-M-036-002-MY3 and 97-EC-17-A-08-S1-006, respectively. We would like to express our gratitude for the use of the facilities of the Core Facilities for Nanoscience and Nanotechnology at Academia Sinica.

\section{References}

[1] T. Seki, T. Shima, K. Yakushiji, K. Takanashi, G.Q. Li, S. Ishio, J. Appl. Phys. 100 (2006) 043915.

[2] C.T. Rettner, M.E. Best, B.D. Terris, IEEE Trans. Magn. 37 (2001) 1649.

[3] K. Naito, H. Hieda, M. Sakurai, Y. Kamata, K. Asakawa, IEEE Trans. Magn. 38 (2002) 1949.
[4] M. Albrecht, G. Hu, I.L. Guhr, T.C. Ulbrich, J. Boneberg, P. Leiderer, G. Schatz, Nat. Mater. 4 (2005) 203.

[5] Z. Huang, Jing Zhu, Appl. Phys. Lett. 91 (2007) 013108.

[6] S.M. Weekes, F.Y. Ogrin, Appl. Phys. Lett. 97 (2007) 10 J503.

[7] C. Kim, T. Loedding, S. Jang, H. Zeng, Z. Li, Y. Sui, D.J. Sellmyer, Appl. Phys. Lett. 91 (2007) 172508.

[8] Y.C. Sui, W. Liu, L.P. Yue, X.Z. Li, J. Zhou, R. Skomski, D.J. Sellmyer, J. Appl. Phys. 97 (2005) 10J304.

[9] K. Liu, J. Nogues, C. Leighton, H. Masuda, K. Nishio, I.V. Roshchin, I.K. Schuller, Appl. Phys. Lett. 81 (2002) 4434.

[10] Y. Lei, W.K. Chim, Chem. Mater. 17 (2005) 580.

[11] M.S. Sanders, A.L. Prieto, R. Gronsky, T. Sands, A.M. Stacy, Adv. Mater. 14 (2002) 665.

[12] M.M. Gonzalez, A.L. Prieto, R. Gronsky, T. Sands, A.M. Stacy, Adv. Mater. 15 (2003) 1003.

[13] H. Wang, Y.C. Wu, L. Zhang, X. Hu, Appl. Phys. Lett. 89 (2006) 232508.

[14] Y. Zhang, G. Li, Y. Wu, B. Zhang, W. Song, L. Zhang, Adv. Mater. 14 (2002) 1227.

[15] S.Z. Chu, S. Inoue, K. Wada, K. Kurashima, Electrochim. Acta 51 (2005) 820.

[16] F.M.F. Rhen, E. Backen, J.M.D. Coey, J. Appl. Phys. 97 (2005) 113908.

[17] Y.H. Huang, H. Okumura, G.C. Hadjipanayis, D. Weller, J. Appl. Phys. 91 (2002) 6869.

[18] C.Y. Kuo, S.Y. Lu, T.Y. Wei, J. Cryst. Growth 285 (2005) 400.

[19] H. Zheng, J. Zhong, Z. Gu, W. Wang, J. Magn. Magn. Mater. 320 (2008) 565.

[20] H. Zheng, J. Zhong, W. Wang, Y. Zheng, C. Ma, Thin Solid Films 516 (2008) 4983.

[21] N. Yasui, A. Imada, T. Den, Appl. Phys. Lett. 83 (2003) 3347.

[22] K. Nielsch, F. Müller, A.P. Li, U. Gosele, Adv. Mater. 12 (2000) 582.

[23] J. Liang, H. Chik, A. Yin, J. Xu, J. Appl. Phys. 91 (2002) 2544.

[24] D. Crouse, Y.H. Lo, A.E. Miller, M. Crouse, Appl. Phys. Lett. 76 (2000) 49

[25] Y.D. Wang, S.J. Chua, M.S. Sander, P. Chen, S. Tripathy, C.G. Fonstad, Appl. Phys. Lett. 85 (2004) 816.

[26] B.D. Cullity, S.R. Stock, Elements of X-ray Diffraction, third edition. Prentice-Hall, 2001.

[27] M. Volmer, A. Weber, Z. Phys. Chem. 119 (1926) 277. 